Revue

Revue de l'histoire des religions

de Ihistoire des religions

3 | 2019

Varia

\title{
Claude CAlame et Pierre ElLINGer (dir.), Du récit au
} rituel par la forme esthétique. Poèmes, images et pragmatique cultuelle en Grèce ancienne

Paris, Les Belles Lettres, 2017

Marie-Joséphine Werlings

\section{OpenEdition}

Journals

Édition électronique

URL : https://journals.openedition.org/rhr/9938

DOI : $10.4000 /$ rhr.9938

ISSN : 2105-2573

Éditeur

Armand Colin

Édition imprimée

Date de publication : 1 septembre 2019

Pagination : 594-597

ISBN : 978-2-200-93260-2

ISSN : 0035-1423

Référence électronique

Marie-Joséphine Werlings, « Claude Calame et Pierre Eluinger (dir.), Du récit au rituel par la forme esthétique. Poèmes, images et pragmatique cultuelle en Grèce ancienne », Revue de I'histoire des religions [En ligne], 3 | 2019, mis en ligne le 01 janvier 2021, consulté le 08 janvier 2022. URL : http:// journals.openedition.org/rhr/9938; DOI : https://doi.org/10.4000/rhr.9938

Ce document a été généré automatiquement le 8 janvier 2022.

Tous droits réservés 


\section{Claude CALAME et Pierre}

ELLINGER (dir.), Du récit au rituel par la forme esthétique. Poèmes, images et pragmatique cultuelle en Grèce

\section{ancienne}

Paris, Les Belles Lettres, 2017

Marie-Joséphine Werlings

\section{RÉFÉRENCE}

Claude CALAME et Pierre ELLINGER (dir.), Du récit au rituel par la forme esthétique. Poèmes, images et pragmatique cultuelle en Grèce ancienne, Paris, Les Belles Lettres, 2017, 21,5 cm, 342 p., 35 €, ISBN 978-2-251-44615-8.

1 Ce livre réunit douze contributions issues d'un colloque international qui s'est tenu à Paris en février 2012, sous l'égide du centre AnHiMa (Anthropologie et histoire des mondes antiques), et qui avait été précédé de trois ateliers et journées d'études. Ces contributions, présentées par une introduction des éditeurs, sont dues aux meilleurs spécialistes contemporains de poésie grecque archaïque et classique (L. Athanassaki, E. Bowie, C.Brillante), de mythologie et religion grecques ( $\mathrm{Cl}$. Calame, P. Ellinger, D. Bouvier, J. N. Bremmer, L. Bruit-Zaidman, V. Pirenne-Delforge et G. Pironti, R.D. Woodard) et d'iconographie grecque (F. Lissarrague). Soulignons tout de suite que l'ampleur du sujet et la variété des cas étudiés ne nuisent en rien à l'homogénéité de ce recueil dont toutes les contributions ont d'ailleurs été rédigées ou traduites en français.

2 Partant du constat que les mythes grecs n'existent pour nous qu'à travers les différentes formes poétiques et iconographiques qui leur ont été données dans des contextes sociaux et cultuels spécifiques et pour des publics déterminés, les 
organisateurs de ces rencontres proposent de renouveler les études de mythologie en abordant les récits mythiques sous cet angle pragmatique - que permet de saisir, par exemple, une analyse de la gestuelle, de la sémantique, de la syntaxe ou encore des images. Il s'agissait autrement dit de dépasser le caractère littéraire figé du récit mythique tel qu'il peut apparaître à première vue en essayant de redonner sens et vie à un "processus symbolique de savoir collectif, de mémoire culturelle et de croyance religieuse dynamique» (p. 8). De ce point de vue, la lecture des mythes grecs proposée dans cet ouvrage s'inscrit dans la lignée des travaux d'anthropologie historique.

Le champ d'investigation proposé est essentiellement celui de la poésie grecque archaïque et classique (Homère, Hésiode, Archiloque, Pindare, Bacchylide, Euripide) et certaines contributions sont l'heureuse occasion de discuter et de faire connaitre des textes difficiles et souvent laissés à l'appréciation de quelques spécialistes. C'est le cas en particulier du poème d'Archiloque consacré à Télèphe ou encore de certains Péans de Pindare, moins connus que ses épinicies, qui nous sont parvenus dans un état très fragmentaire et qui trouvent ici un nouvel éclairage bienvenu, sous la plume de $\mathrm{Cl}$. Calame et de E. Bowie en particulier. Mais l'intérêt de la réflexion proposée tient par ailleurs au fait qu'elle aborde aussi la question sous un angle diachronique en s'intéressant même aux périodes plus tardives (jusqu'à Clément d'Alexandrie) où l'insertion du mythe dans un rituel a perdu de son actualité religieuse et tend à devenir un héritage culturel offert à la curiosité et à la soif de savoir de l'auditoire.

4 La problématique de l'ouvrage fait bien entendu une part essentielle à l'étude des rapports entre le mythe et le rite. La question n'est certes pas neuve et aucun des auteurs n'ignore les dangers d'une telle approche qui risque toujours de tourner à l'utilisation de "concepts opératoires" appartenant bien plus au monde de l'anthropologue contemporain qu'à celui du poète grec de l'Antiquité. J. Bremmer met d'ailleurs en garde contre la naïveté qu'il y aurait à croire que l'étude des interactions entre mythe et rite serait la clé unique d'accès à l'un et l'autre. En relisant le récit héroïque de l'éducation de Thésée transmis par Plutarque au tout début de l'ère chrétienne, il suggère que mythe et rite ont pu avoir leur développement propre, quitte à ce que certains aspects du rituel soient $a$ posteriori rattachés au mythe et justifiés par lui.

5 Ce qui frappe, à la lecture de ce recueil, est de constater à quel point les notions de « rite » ou de "pratiques rituelles » renvoient à des situations très variées. Si, par exemple, Cl.Calame, L. Athanassaki ou P. Ellinger s'intéressent au choix et au développement de l'épisode mythologique dans le cadre des différentes manifestations religieuses célébrant les athlètes victorieux aux jeux panhelléniques, L. Bruit Zaidman montre comment Euripide, à travers sa tragédie Iphigénie en Tauride, propose à ses contemporains une réflexion sur l'héritage des mythes héroïques anciens et leur inscription dans les rituels contemporains autour d'une question particulièrement difficile à comprendre, celle du sacrifice humain. C. Brillante, pour sa part, à travers l'étude des traditions poétiques concernant les Muses, suggère l'existence d'un culte en leur honneur sur l'Hélicon dès avant la composition des poèmes d'Hésiode. Quant à E. Bowie et F. Lissarrague, ils s'intéressent à la production de récits et d'images mythologiques dans le contexte rituel du banquet.

6 C'est finalement le concept même de rituel qui est poussé à ses limites dans la brillante contribution de G. Pironti et V. Pirenne Delforge, qui montrent comment le sophiste alexandrin Philostrate, rapportant une prétendue description d'un tableau censé 
représenter un chœur de jeunes filles célébrant Aphrodite, recompose en réalité par son discours un univers rituel tout à fait original. Il ne s'agit en effet vraisemblablement pas de la représentation d'un culte précis car les références explicites et implicites au mythe d'Aphrodite savamment tissées dans cette ekphrasis virtuose tendent plutôt à dessiner une image "panhellénique" d'Aphrodite « recomposée dans une savante mosaïque à l'aide de tessons choisis qu[e l'auteur] puise à la sculpture, à la poésie mélique, à l'épopée archaïque, et ainsi de suite. L'ancrage rituel de ces différents langages constitue la base sur laquelle s'appuie et devient crédible la transfiguration de cette mosaïque en performance chorale en l'honneur de la déesse » (p. 254).

7 Mais ce recueil parvient à dépasser habilement la difficile question des rapports entre mythe et rite en orientant la réflexion sur le statut de la parole poétique en contexte cultuel. La notion de "contextualisation" du récit mythique est en effet au cœur de chaque contribution. Dans celles consacrées aux épinicies de Pindare ou de Bacchylide (Cl. Calame, L. Athanassaki ou P. Ellinger en particulier), il s'agit d'étudier comment la mise en mot du mythe et du rite au sein duquel il est énoncé repose sur les liens tissés par le poète entre l'épisode mythologique choisi et le lieu de la performance (Delphes ou la patrie du vainqueur, par exemple). Ainsi que le rappelle L. Athanassaki, les indications concernant le contexte rituel de la performance sont nombreuses dans les épinicies, d'une part car elles étaient absolument indispensables pour préserver la mémoire de ce contexte rituel original dans une société qui ne disposait d'aucun moyen moderne d'enregistrement audio ou vidéo et d'autre part car elles participaient aussi à la création d'une « attitude émotionnelle positive » (p. 79) envers l'athlète célébré. C'est aussi parfois le poème mettant en forme le mythe qui apparaît lui-même comme une composante essentielle du culte : D. Bouvier montre bien que, dans l'esprit des poètes archaïques, la performance poétique constituait effectivement, pour reprendre une image pindarique à laquelle il redonne tout son sens, une « offrande sans fumée ». Voilà qui finit de brouiller les distinctions qu'on aura voulu établir entre le mythe expliquant le rite et le rite mettant en action le mythe.

8 Mais le contexte de la parole poétique racontant le mythe dans le cadre d'un rituel ne se réduisait pas, pour les Anciens, à l'environnement immédiat (lieu de la performance, public présent, paroles prononcées, gestes accomplis), il englobait aussi l'ensemble des manifestations cultuelles associées. D. Woodard démontre ainsi qu'un épisode hérö̈que du mythe de Bellérophon rapporté par Plutarque a correspondu à de très anciens rituels de réintégration du guerrier dans sa communauté. Une fois ces rituels tombés en désuétude, ce mythe, dont les schémas fondamentaux ont de nombreuses correspondances dans d'autres mythes indo-européens, est resté figé dans sa forme littéraire. Seul l'ensemble cultuel dans lequel est inséré l'espace dédié au culte héroïque de Bellérophon à Corinthe préserve le contexte religieux dans lequel le mythe s'est déployé, sans doute avant l'époque archaïque, dès l'époque mycénienne.

Par l'attention portée à la mise en forme et à la contextualisation du mythe, cet ouvrage répond donc finalement à la fascination que les modernes ont toujours eue pour les mythes grecs et leurs infinies variations formelles et narratives. Les multiples formes qu'adoptent les mythes ne sont sans aucun doute pas uniquement le fruit du génie poétique, ni des joutes littéraires et artistiques encourageant l'imagination. Dans la mise en mot et en image du mythe se jouait chaque fois, à travers le contexte rituel, une réactualisation unique du passé héroïque. 


\section{AUTEURS}

MARIE-JOSÉPHINE WERLINGS

Université Paris Nanterre. 